\title{
Better clinical outcome with direct oral anticoagulants in hospitalized heart failure patients with atrial fibrillation
}

\author{
Akiomi Yoshihisa ${ }^{*}$, Yu Sato, Takamasa Sato, Satoshi Suzuki, Masayoshi Oikawa and Yasuchika Takeishi
}

\begin{abstract}
Background: Atrial fibrillation (AF) is common in patients with heart failure and is associated with higher mortality. Although previous studies have reported that direct oral anticoagulants (DOACs) reduce the risk of cardiovascular events in out-patients with AF, it remains unclear whether DOACs reduce mortality in hospitalized heart failure (HHF) patients with AF. Therefore, we examined the impact of DOACs on mortality in this group of patients.

Methods: Consecutive $497 \mathrm{HHF}$ patients with AF were retrospectively registered and divided into three groups on the basis of the presence of anticoagulant therapy: non-anticoagulant group (Non, $n=90$ ), Vit K antagonists (VKAs) group $(n=257)$ and DOACs group $(n=150)$. We followed up all the patients for mortality.

Results: In the Kaplan-Meier analysis (mean follow-up of 1093 days), all-cause mortality was significantly lower in the VKAs and DOACs groups than in the Non group (31.1\% and $15.3 \%$ vs. $43.3 \%$, log-rank $P<0.001)$. In the multivariable Cox proportional hazard analysis after adjusting for other potential confounding factors, usage of DOACs and VKAs were independently associated with lower mortality in HHF patients AF (DOACs, HR 0.356, P=0.001; VKAs, HR 0.472, $P=0.002$ ). Furthermore, the propensity-matched 1:1 cohort was assessed based on the propensity score (DOACs, $n=114$ and VKAs, $n=114$ ). All-cause mortality was significantly lower in the DOACs group than in the VKAs group in the post-matched cohort $(12.3 \%$ vs. $35.1 \%$, log-rank $P=0.038)$. In the Cox proportional hazard analysis, the use of DOACs was associated with lower mortality in the post-matched cohort (HR 0.526, $P=0.041$ ).
\end{abstract}

Conclusion: Appropriate use of anticoagulants in HHF patients with AF is important, and DOACs potentially improve all-cause mortality in such patients.

Keywords: Heart failure, Atrial fibrillation, Anticoagulant therapy, Direct oral anticoagulants, Vitamin K antagonists, Mortality

\section{Background}

Heart failure (HF) is a systemic disease with a devastating prognosis, which affects many organ systems, including the cardiovascular system. In HF patients, atrial fibrillation (AF) is a frequent co-morbidity and its prevalence is related to the severity of the clinical status of patients [1]. HF and AF share common risk factors and clinical backgrounds (e.g. inflammation and fibrosis), and the occurrence of either of them may induce the onset of a vicious circle which, in turn, facilitates the manifestation of the other [2-4]. AF subsequently causes stroke and/or HF [5]. On the other hand, the incidence

\footnotetext{
* Correspondence: yoshihis@fmu.ac.jp

Department of Cardiovascular Medicine, Fukushima Medical University, 1 Hikarigaoka, Fukushima 960-1295, Japan
}

of AF increases with severity of HF [4], and AF in HF patients is strongly associated with all-cause mortality including sudden cardiac death [6] and bleeding, rather than stroke or embolism [7-9]. Hence, all-cause mortality is the most meaningful endpoint in such patients.

Anticoagulant therapy is prescribed in consideration of balance of efficacy for embolic risk (e.g. CHADS score, $\mathrm{CHA}_{2} \mathrm{DS}_{2}$-VASc score) and safety for bleeding risk (e.g. HAS-BLED score) in AF patients [10]. Direct oral anticoagulants (DOACs) have the potential to reduce the burden of stroke as effective, safe, and more convenient alternatives to vitamin $\mathrm{K}$ antagonists (VKAs), such as warfarin [11-13]. A recent meta-analysis of randomized controlled studies revealed that DOACs significantly reduce the risk of stroke or systemic embolic events by 
$19 \%$ and hemorrhagic stroke by $51 \%$ compared with VKAs, but increased the risk of gastrointestinal hemorrhage by $25 \%$ in out-patients with AF [12]. On the other hand, a few post-hoc analyses limited to AF outpatients with HF revealed that 1) DOACs were similarly effective or safer (less intracranial hemorrhage) compared to VKA in AF out-patients with HF compared with those without HF, and that 2) DOACs have not yet improved all-cause mortality of AF out-patients with HF $[7-9,14]$. However, there were several problems in the previous studies [7-9, 14]: 1) out-patients with reduced left ventricular ejection fraction and no symptoms of HF were included in previous studies, $[7-9,14]$ and hospitalized heart failure (HHF) patients had higher accurate diagnosis of HF and risk of mortality than out-patients [15-17], 2) important factors for HF, such as Framingham criteria, etiology, presence of anemia and hyponatremia, natriuretic peptide, medications of HF, were partly absent or were not considered in these post-hoc analyses $[7-9,14], 3)$ anticoagulant therapy could not be used in all the patients with HF and AF in a real world setting [18] because of certain contraindications for anticoagulants (higher HAS-BLED score and presence of anemia) [18]. Additionally, studies on symptomatic HHF patient coexistence with AF have not been previously reported. The association between DOACs and all-cause mortality in HHF patients with AF is still unclear and controversial. Therefore, we examined the impact of anticoagulant therapy on all-cause mortality in symptomatic HHF patients with $\mathrm{AF}$ based on a retrospective study, using propensity score (PS) analyses to reduce selection bias, and taking into consideration the patients' clinical backgrounds, including $\mathrm{CHA}_{2} \mathrm{DS}_{2}$-Vasc and HAS-BLED scores, other co-morbidities and pharmacotherapies.

\section{Methods \\ Subjects and study protocol}

This was a observational study analyzed using PS methods in which we enrolled consecutive HHF patients with AF, who were hospitalized with decompensated HF defined based on the Framingham criteria [19]., and discharged from Fukushima Medical University Hospital between 2011 and 2015. AF was identified by an electrocardiogram performed during hospitalization and/or medical records including past history. The patient flow chart is shown in Fig. 1. In HHF patients with AF $(n=627)$, patients with severe valvular etiology and/or post-operative state $(n=75)$, dialysis and/or creatinine clearance less than $30 \mathrm{ml} / \mathrm{min}(n=43)$, acute coronary syndrome $(n=6)$, and documented advanced cancer $(n=6)$ were excluded, and 497 HHF patients with AF were finally enrolled. Patients were divided into three groups on the basis of the use of VKAs and DOACs at hospital discharge: non-anticoagulant group (Non, $n=90$ ), VKAs group (warfarin, $n=257)$ and DOACs group $(n=150)$. The DOACs used in this study were as follows: apixaban $(n=52$, $34.7 \%)$, rivaroxaban $(n=35,23.3 \%)$, edoxaban $(n=33$, $22.0 \%)$ and dabigatran $(n=30,20.0 \%)$. We compared the clinical features and laboratory data that were recorded at hospital discharge. The CHADS score, $\mathrm{CHA}_{2} \mathrm{DS}_{2}$-VASc score, HAS-BLED score, and time in therapeutic range (TTR) of VKAs were measured as previously reported [10]. The left ventricular ejection fraction (LVEF) was calculated using Simpson's

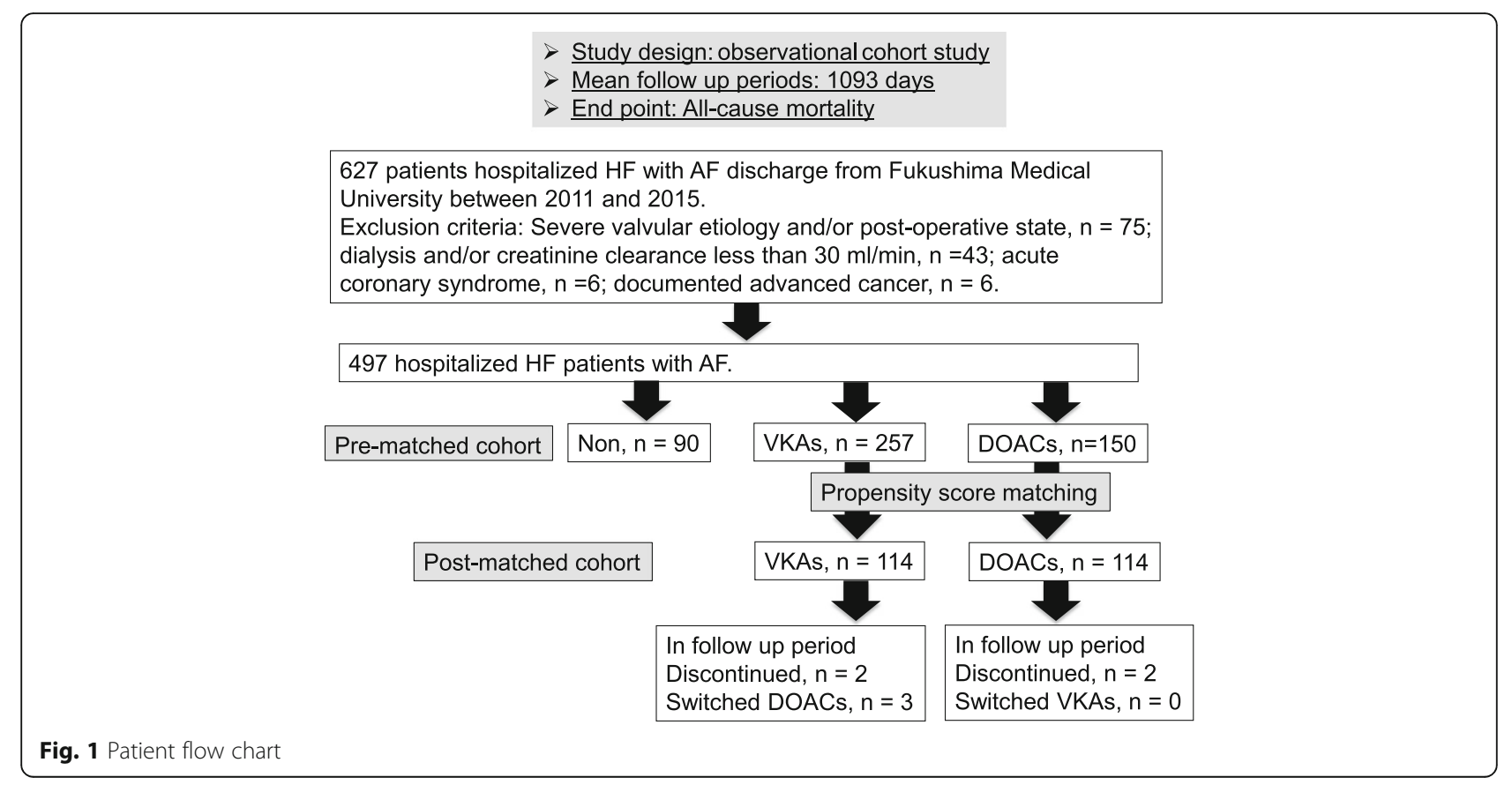


method, and recordings were performed on ultrasound systems (ACUSON Sequoia, Siemens Medical Solutions USA, Inc., Mountain View, CA, USA). Preserved LVEF was defined as more than LVEF $50 \%$. All patients were followed up until 2017 for all-cause mortality. Cardiac death was defined by experienced cardiologists as death due to worsening HF in accordance with the Framingham criteria [19], due to ventricular fibrillation determined by electrocardiogram or implantable devices, and acute myocardial infarction. Survival time was calculated from the day of discharge to the date of death or last follow-up. Status and dates of deaths were checked from medical recoreds of the patients. If these data were unavailable, status was examined by a telephone call to the patient's referring hospital physicians. The end-point classification committee, comprising two experienced cardiologists who were not study investigators, reviewed the data. We could follow up all the patients.

Written informed consent was obtained from each subject, and the study protocol was approved by the ethics committee of Fukushima Medical University (No. 823). The investigation conforms with the principles outlined in the Declaration of Helsinki. Reporting of the study conforms to STROBE along with references to STROBE and the broader EQUATOR guidelines [20].

\section{Statistical analysis}

Categorical variables are expressed as numbers and percentages, and the chi-square test was used for their comparisons. Normally distributed data are presented as mean \pm SD, and non-normally distributed data are presented as median and interquartile range, or logtransformed. Data among the three groups were compared using analysis of variance followed by Tukey's post-hoc test. The Kaplan-Meier method was used for presenting the mortality, and the log-rank test was performed.

To eliminate imbalances in the measurement of baseline characteristics because of selection bias, we used multiple approaches, including multiple Cox regression analysis in the pre-matched cohort $(n=497)$ and PS matching in the post-matched cohort $(n=228)$. To prepare for potential confounding in the Cox regression analyses, we considered the following clinical factors, which are known to affect the mortality in HF patients: age, sex, B-type natriuretic peptide (BNP), presence of New York Heart Association functional class III or IV, ischemic etiology, preserved LVEF, hypertension, diabetes, CKD, anemia, hyponatremia (as sodium less than $135 \mathrm{mEq} / \mathrm{l}$ ), and oral administration of renin-angiotensin-aldosterone system inhibitors, $\beta$-blockers, diuretics, inotropic agents, and anticoagulants. These factors, which predicting mortality with value of $P<0.05$, were selected in the final adjusted model.

Furthermore, the PS for treatment with DOAC was estimated for each patient by logistic regression with the following variables, which are important considerable factors to prescribe DOACs: age, body weight, $\mathrm{CHA}_{2} \mathrm{DS}_{2}$-Vasc score, HAS-BLED score and creatinine clearance. The PS is the propensity from 0 to 1 to receive treatment, and is used to adjust potential selection bias and confounding factors between the groups with or without treatment in the observational studies [21]. We used the PS to match patients who were administered DOAC and those who were administered VKA, using 1:1 nearest neighbor matching algorithm and a 0.2 caliper width of the standard deviation of the PS logit with caliper as of 0.03 [22]. The PS-matched datasets were compared using a pairwise analysis [23], and the postmatched cohort (DOACs, $n=114$ and VKAs, $n=114$ ) was defined. To assess potential heterogeneity of the effect of DOAC on mortality, we conducted subgroup analyses in the post-matched cohort $(n=228)$. We tested for first order interactions using multivariable Cox proportional hazard models by entering interaction terms between DOAC use and the subgroup variables. Missing values were handled by estimating one logistic regression model for each pattern of missing values. A $P$ value of $<0.05$ was considered significant for all comparisons. These analyses were performed using a statistical software package (SPSS ver. 24.0, IBM, Armonk, NY, USA).

\section{Results}

The clinical features of the study subjects are presented in Table 1. The Non group had the highest age, HAS-BLED score, prevalence of paroxysmal AF, female gender and anemia. It seems that this background is associated with abandonment of anticoagulants. The VKAs group had the highest prevalence of male gender and hypertension, and highest usage of $\beta$-blockers, diuretics, and inotropic agents. In addition, TTR was $70 \%$ in VKAs group (data not shown in Table 1). The DOACs group had the lowest prevalence of anemia. In contrast, $\mathrm{CHADS}_{2}$ and $\mathrm{CHA}_{2} \mathrm{DS}_{2}$-Vasc scores, prevalence of NYHA class III or IV, preserved LVEF, other co-morbidities, BNP, $\mathrm{C}$-reactive protein and sodium did not significantly differ among the three groups.

In the follow-up period (mean of 1093 days), 70 cardiac deaths (worsened HF $n=42$, ventricular fibrillation $n=22$ and acute coronary syndrome $n=6$ ) and 72 non-cardiac deaths (stroke $n=10$, gastrointestinal hemorrhage $n=7$, aneurysms $n=5$, trauma $n=4$, pneumonia/respiratory failure/hemorrhage $n=18$, cancer $n=12$, infection/disseminated intravascular 
Table 1 Comparisons of clinical features $(n=497)$

\begin{tabular}{|c|c|c|c|c|}
\hline & Non $(n=90)$ & VKAs $(n=257)$ & DOACs $(n=150)$ & $P$ value \\
\hline Age (years) & $74.4 \pm 12.0$ & $69.9 \pm 11.8^{* *}$ & $70.4 \pm 12.9^{*}$ & 0.010 \\
\hline $\mathrm{CHADS}_{2}$ score & $3.2 \pm 1.3$ & $3.1 \pm 1.2$ & $2.9 \pm 1.3$ & 0.286 \\
\hline $\mathrm{CHA}_{2} \mathrm{DS}_{2}$-Vasc score & $4.8 \pm 1.6$ & $4.4 \pm 1.7$ & $4.3 \pm 1.8$ & 0.074 \\
\hline HAS-BLED score & $3.4 \pm 1.3$ & $2.8 \pm 1.2^{* *}$ & $2.7 \pm 1.4^{*}$ & 0.006 \\
\hline Paroxysmal af (n, \%) & $44(48.9)$ & 89 (34.6) & $52(34.7)$ & 0.041 \\
\hline Male gender (n, \%) & $44(48.9)$ & $175(68.1)$ & $89(59.3)$ & 0.004 \\
\hline Body mass index $\left(\mathrm{kg} / \mathrm{cm}^{2}\right)$ & $22.7 \pm 4.9$ & $23.3 \pm 4.0$ & $23.5 \pm 3.9$ & 0.404 \\
\hline Systolic BP (mmHg) & $130.8 \pm 33.1$ & $123.8 \pm 28.8$ & $132.3 \pm 36.3$ & 0.021 \\
\hline Diastolic BP (mmHg) & $72.8 \pm 19.2$ & $72.6 \pm 18.6$ & $76.0 \pm 24.3$ & 0.265 \\
\hline Heart rate (bpm) & $89.7 \pm 35.0$ & $84.1 \pm 29.1$ & $85.5 \pm 35.8$ & 0.371 \\
\hline NYHA class III or IV (n, \%) & $6(6.7)$ & $8(3.1)$ & $4(2.7)$ & 0.226 \\
\hline Preserved LVEF $(n, \%)$ & $41(45.6)$ & $126(49.0)$ & $67(44.7)$ & 0.662 \\
\hline Etiology & & & & 0.130 \\
\hline Ischemic (n, \%) & $26(28.9)$ & $68(26.5)$ & $43(28.7)$ & \\
\hline Cardiomyopathy (n, \%) & $23(25.6)$ & $68(26.5)$ & $36(24.0)$ & \\
\hline Valvular (n, \%) & $19(21.1)$ & $70(27.2)$ & $25(16.7)$ & \\
\hline Others $(n, \%)$ & $22(24.4)$ & $51(19.8)$ & $46(30.7)$ & \\
\hline \multicolumn{5}{|l|}{ Co-morbidity } \\
\hline Hypertension (n, \%) & $67(74.4)$ & $202(78.6)$ & $98(65.3)$ & 0.013 \\
\hline Diabetes (n, \%) & $36(40.0)$ & $103(40.1)$ & $53(35.3)$ & 0.611 \\
\hline Dyslipidemia (n, \%) & $66(73.3)$ & $185(72.0)$ & $97(64.7)$ & 0.224 \\
\hline CKD $(n, \%)$ & $49(54.4)$ & $163(63.4)$ & $85(56.7)$ & 0.213 \\
\hline Anemia (n, \%) & $58(64.4)$ & $144(56.0)$ & $70(46.7)$ & 0.023 \\
\hline Stroke $(n, \%)$ & $23(25.6)$ & $65(25.3)$ & $32(21.3)$ & 0.628 \\
\hline \multicolumn{5}{|l|}{ Medications } \\
\hline RAS inhibitors (n, \%) & $61(67.8)$ & $206(80.2)$ & $115(76.7)$ & 0.057 \\
\hline$\beta$-blockers (n, \%) & $63(70.0)$ & $219(85.2)$ & $116(77.3)$ & 0.005 \\
\hline Diuretics (n, \%) & $55(61.1)$ & $214(83.3)$ & $103(68.7)$ & $<0.001$ \\
\hline Inotropic agents (n, \%) & $9(10.0)$ & $52(20.2)$ & $7(4.7)$ & $<0.001$ \\
\hline Antiplatelet agents (n, \%) & $43(47.8)$ & $108(42.0)$ & $62(41.3)$ & 0.576 \\
\hline \multicolumn{5}{|l|}{ Laboratory data } \\
\hline BNP $(p g / m l) \S$ & $332.8(140.3-656.1)$ & $284.1(157.4-625.9)$ & $270.8(101.8-552.2)$ & 0.494 \\
\hline C-reactive protein $(\mathrm{mg} / \mathrm{dl}) \S$ & $0.13(0.05-0.53)$ & $0.07(0.03-0.18)$ & $0.08(0.04-0.25)$ & 0.104 \\
\hline Sodium (mEq/l) & $138.6 \pm 5.1$ & $138.7 \pm 4.0$ & $139.2 \pm 4.3$ & 0.561 \\
\hline
\end{tabular}

Af atrial fibrillation, BP blood pressure, NYHA New York Heart Association, LVEF left ventricular ejection fraction, CKD chronic kidney disease, RAS rennin-angiotensinaldosterone system; $B N P$ B-type natriuretic peptide

${ }^{*} P<0.05$ and ${ }^{*} P<0.01$ vs. Non group

$\S D a t a$ are presented as median (interquartile range)

coagulation $n=5$, systemic embolism, $n=3$, renal failure $n=3$, others $n=5$ ) occurred. As shown in Fig. 2, allcause mortality was significantly lower in the DOACs and VKAs groups than in the Non group in the prematched cohort (Fig. 2; $P<0.001$ ). In the Cox proportional hazard analysis after adjusting for potential confounding factors (Table 2), the usage of DOACs and
VKAs was independently associated with lower mortality in HHF patients with AF (DOACs, HR 0.356, $P=0.001$; VKAs, HR 0.472, $P=0.002$ ).

In addition, in the post-matched cohort, mortality was significantly lower in the DOACs group than in the VKAs group (Fig. 3; $P=0.038$ ). The clinical features of DOACs group and VKAs group in the post-matched 


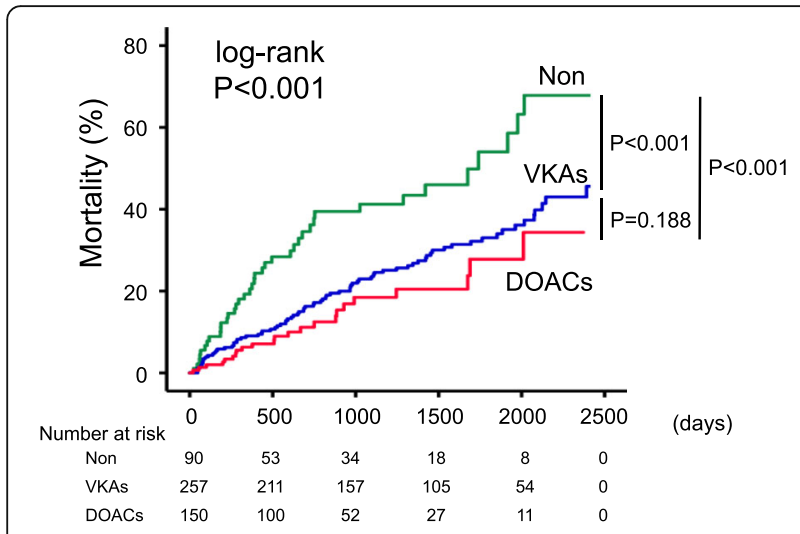

Fig. 2 All-cause mortality: pre-matched cohort. Kaplan-Meier analyses for all-cause mortality among the three groups (Non group, $n=90$; VKAs group, $n=257$; DOACs group, $n=150$ ) in the pre-matched cohort $(n=497)$;

cohort are summarized in Additional file 1: Table S1. Interactions between the DOACs group and clinically relevant variables were modeled with the Cox regression analysis, as shown in Table 3, for all-cause mortality in the post-matched cohort $(n=228)$. In the Cox proportional hazard analysis (Table 3), the usage of DOACs was associated with lower mortality in the post-matched cohort (HR 0.526, 95\% CI 0.284-0.974, $P=0.041$ ). There were no interactions between DOACs use and other

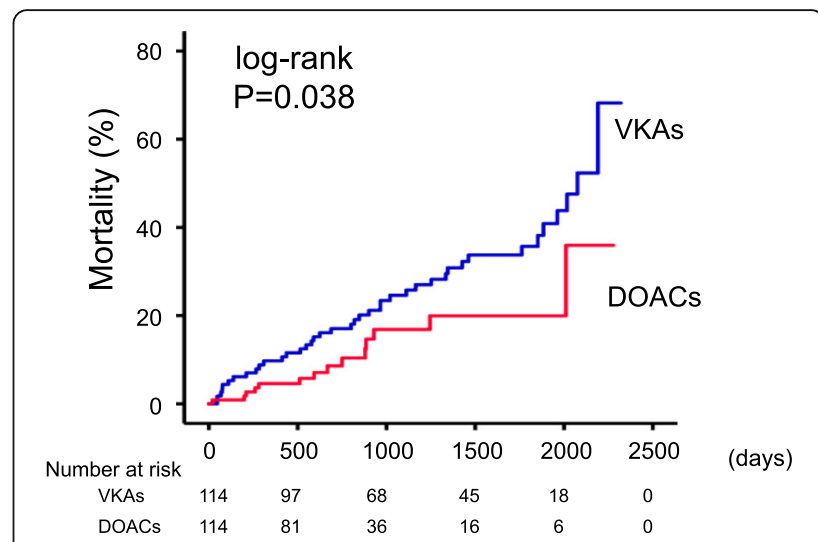

Fig. 3 All-cause mortality: post-matched cohort. Kaplan-Meier analyses for all-cause mortality between the groups (VKAs group, $n=114$ and DOACs group, $n=114)$ in the post-matched cohort $(n=228)$

important variables (e.g. CKD, anemia, LVEF, other pharmacotherapies) that affected mortality in all subgroups.

\section{Discussion}

To the best of our knowledge, the present study is the first to show the association between DOACs and lower all-cause mortality in HHF patients with AF based on a real world observational study using multiple Cox regression and PS analyses, considering clinical backgrounds, including $\mathrm{CHA}_{2} \mathrm{DS}_{2}$-Vasc and

Table 2 Cox proportional hazard model of all-cause mortality (event $=142 / n=497$ )

\begin{tabular}{|c|c|c|c|c|c|c|}
\hline \multirow[t]{2}{*}{ Risk factor } & \multicolumn{3}{|c|}{ Univariable } & \multicolumn{3}{|c|}{ Multivariable } \\
\hline & $\mathrm{HR}$ & $95 \% \mathrm{Cl}$ & $P$ value & $\mathrm{HR}$ & $95 \% \mathrm{Cl}$ & $P$ value \\
\hline Age & 1.045 & $1.028-1.063$ & $<0.001$ & 1.025 & $1.007-1.044$ & 0.007 \\
\hline Male sex & 1.044 & $0.741-1.472$ & 0.805 & & & \\
\hline NYHA class III or IV & 4.662 & $2.718-7.995$ & $<0.001$ & 1.561 & $0.826-2.949$ & 0.170 \\
\hline Ischemic etiology & 1.031 & $0.682-1.560$ & 0.885 & & & \\
\hline Preserved LVEF & 0.770 & $0.552-1.073$ & 0.122 & & & \\
\hline Hypertension & 1.089 & $0.719-1.651$ & 0.687 & & & \\
\hline Diabetes & 1.331 & $0.957-1.851$ & 0.089 & & & \\
\hline Chronic kidney disease & 1.842 & $1.274-2.664$ & 0.001 & 1.352 & $0.899-2.032$ & 0.147 \\
\hline Anemia & 2.456 & $1.688-3.573$ & $<0.001$ & 1.849 & $1.214-2.816$ & 0.004 \\
\hline Hyponatremia & 2.089 & $1.346-3.241$ & 0.001 & 1.406 & $0.890-2.222$ & 0.144 \\
\hline Log BNP & 3.541 & $2.394-5.238$ & $<0.001$ & 2.970 & $1.903-4.635$ & $<0.001$ \\
\hline RAS inhibitors & 0.632 & $0.433-0.921$ & 0.017 & 0.750 & $0.492-1.141$ & 0.179 \\
\hline$\beta$-blockers & 0.636 & $0.437-0.927$ & 0.018 & 0.657 & $0.426-1.014$ & 0.058 \\
\hline Diuretics & 2.290 & $1.379-3.802$ & 0.001 & 2.008 & $1.137-3.546$ & 0.016 \\
\hline Inotropic agents & 2.026 & $1.377-2.982$ & $<0.001$ & 2.126 & $1.366-2.308$ & 0.001 \\
\hline Anticoagulants: Non & Ref & & & Ref & & \\
\hline VKAs & 0.493 & $0.335-0.725$ & $<0.001$ & 0.472 & $0.296-0.750$ & 0.002 \\
\hline DOACs & 0.352 & $0.210-0.590$ & $<0.001$ & 0.356 & $0.199-0.638$ & 0.001 \\
\hline
\end{tabular}


Table 3 Subgroup analysis for all-cause mortality: DOACs vs. VKAs use in post matched cohort

\begin{tabular}{|c|c|c|c|c|c|c|}
\hline Factor & Subgroup & $n$ & $\mathrm{HR}$ & $95 \% \mathrm{Cl}$ & $P$ value & Interaction $P$ value \\
\hline DOACs vs. VKAs & Total & 228 & 0.526 & $0.284-0.974$ & 0.041 & - \\
\hline \multirow[t]{2}{*}{ Age } & $\geq 70$ & 126 & 0.604 & $0.299-1.219$ & 0.159 & 0.537 \\
\hline & $<70$ & 102 & 0.331 & $0.091-1.206$ & 0.094 & \\
\hline \multirow[t]{2}{*}{ Sex } & Male & 159 & 0.569 & $0.287-1.127$ & 0.106 & 0.809 \\
\hline & Female & 69 & 0.539 & $0.108-2.685$ & 0.451 & \\
\hline \multirow[t]{2}{*}{ NYHA class } & ।, II & 222 & 0.561 & $0.301-1.044$ & 0.068 & 0.978 \\
\hline & III, IV & 6 & 0.024 & $0.000-397.4$ & 0.540 & \\
\hline \multirow[t]{2}{*}{ BNP } & >median & 114 & 0.341 & $0.115-1.012$ & 0.053 & 0.168 \\
\hline & $<$ median & 114 & 0.690 & $0.319-1.489$ & 0.344 & \\
\hline \multirow[t]{2}{*}{ LVEF } & Reduced & 127 & 0.676 & $0.312-1.465$ & 0.321 & 0.245 \\
\hline & Preserved & 101 & 0.366 & $0.132-1.014$ & 0.053 & \\
\hline \multirow[t]{2}{*}{ Ischemic etiology } & Present & 63 & 0.926 & $0.319-2.002$ & 0.466 & 0.269 \\
\hline & Absent & 165 & 0.435 & $0.207-0.915$ & 0.028 & \\
\hline \multirow[t]{2}{*}{ Diabetes } & Present & 92 & 0.693 & $0.302-1.594$ & 0.388 & 0.364 \\
\hline & Absent & 136 & 0.401 & $0.159-1.011$ & 0.053 & \\
\hline \multirow[t]{2}{*}{ CKD } & Present & 131 & 0.598 & $0.302-1.185$ & 0.141 & 0.994 \\
\hline & Absent & 97 & 0.667 & $0.152-2.931$ & 0.591 & \\
\hline \multirow[t]{2}{*}{ Anemia } & Present & 106 & 0.474 & $0.215-1.044$ & 0.064 & 0.498 \\
\hline & Absent & 122 & 0.729 & $0.251-2.112$ & 0.560 & \\
\hline \multirow[t]{2}{*}{ RAS inhibitors } & Present & 188 & 0.664 & $0.336-1.313$ & 0.239 & 0.171 \\
\hline & Absent & 40 & 0.187 & $0.041-0.861$ & 0.031 & \\
\hline \multirow[t]{2}{*}{$\beta$-blockers } & Present & 186 & 0.564 & $0.288-1.106$ & 0.096 & 0.389 \\
\hline & Absent & 42 & 0.293 & $0.061-1.394$ & 0.123 & \\
\hline \multirow[t]{2}{*}{ Diuretics } & Present & 166 & 0.630 & $0.333-1.191$ & 0.155 & 0.475 \\
\hline & Absent & 62 & 0.452 & $0.041-4.992$ & 0.517 & \\
\hline \multirow[t]{2}{*}{ Antiplatelet agents } & Present & 97 & 0.863 & $0.386-1.931$ & 0.720 & 0.117 \\
\hline & Absent & 131 & 0.295 & $0.109-0.796$ & 0.016 & \\
\hline
\end{tabular}

DOACs direct oral anticoagulants, VKAs Vit K antagonists, NYHA New York Heart Association, BNP B-type natriuretic peptide, LVEF left ventricular ejection fraction, $C K D$ chronic kidney disease, RAS rennin-angiotensin-aldosterone system

HAS-BLED scores, other co-morbidities, and pharmacotherapies.

To improve the prognosis of HHF patients with AF, prevention of stroke and systemic embolism, as well as avoidance of major bleeding, may be the therapeutic target. To this point, appropriate use of DOACs is expected to be associated with better prognosis in $\mathrm{HHF}$ patients with AF. There are several randomized clinical trials in out-patients with AF regarding efficacy (prevention of stroke and/or systemic embolism) and safety (avoidance of intra cranial hemorrhage or gastrointestinal hemorrhage) of DOACs compared with VKAs. Firstly, dabigatran tended to lower all-cause mortality (RR 0.88, 95\% CI 0.77-1.00) in the RE-LY trial, with enrolled 18,113 out-patients with $\mathrm{AF}\left(\mathrm{CHADS}_{2}\right.$ score $=2.1$, $\mathrm{TTR}=67 \%$, HF patients $32 \%$ ) [24]. In the post-hoc analysis, the relative effects of dabigatran, compared to VKAs, on the occurrence of stroke or systemic embolism and major bleeding were consistent among those with or without HF and those with reduced or preserved LVEF [7]. Secondly, rivaroxaban tended to lower all-cause mortality (RR $0.85,95 \%$ CI $0.70-1.02$ ) in the ROCKET-AF trial, with 14,264 enrolled out-patients with $\mathrm{AF}\left(\mathrm{CHADS}_{2}\right.$ score $=3.5$, TTR $=58 \%$, HF patients $63.7 \%$ ) [25]. In the post-hoc analyses, the efficacy of rivaroxaban was similar in AF out-patients with or without HF [9]. Among the AF patients with HF, the efficacy of rivaroxaban was similar, irrespective of NYHA class, $\mathrm{CHADS}_{2}$ score, and LVEF [9]. Thirdly, apixaban significantly decreased all-cause mortality (RR 0.89, 95\% CI 0.80-0.998) in the ARISTOTLE trial, with 18,201 enrolled out-patients with $\mathrm{AF}\left(\mathrm{CHADS}_{2}\right.$ score $=2.1$, TTR $=66 \%$, HF patients $35 \%$ ) [26]. In the post-hoc analyses, apixaban reduced the risk of stroke, systemic embolism or all-cause death, irrespective of the presence of $\mathrm{HF}$ and/or reduced LVEF [8]. Fourthly, edoxaban 
decreased cardiovascular mortality (RR $0.87,95 \% \mathrm{CI}$ 0.78-0.96) in the ENGAGE-AF TIMI 48, with 21,105 enrolled out-patients with AF $\left(\mathrm{CHADS}_{2}\right.$ score $=2.8$, TTR $=68 \%$, HF patients 58\%) [27]. Although these previous post-hoc analyses [7-9] are partially concordant with our results, detailed data of HF, such as Framingham criteria, etiology of HF, natriuretic peptide and other co-morbidities, were unknown unlike in our results. Furthermore, there is no report regarding efficacy of DOACs on mortality in HHF patients with AF.

In previous studies regarding DOACs compared to VKAs in AF out-patients with HF, 57\% took ACE inhibitors and $68 \%$ took $\beta$ blockers in the RE-LY trial [7], and $60 \%$ took ACE inhibitors and $69 \%$ took $\beta$ blockers in the ROCKET AF trial, in which rivaroxaban tended to decrease all-cause mortality (RR $0.93,95 \%$ CI $0.82-1.07$ ) [9]. In the ARISTOTLE trial, 71\% took ACE inhibitors and $71 \%$ took $\beta$ blockers; however, apixaban did not decrease mortality (reduced LVEF, RR 0.98, 95\% CI 0.79-1.21; preserved LVEF, RR 0.89 , 95\% CI 0.69-1.13) [8]. Our study subjects were HHF, and had a relatively higher $\mathrm{CHADS}_{2}$ score of 3.1 , TTR of $70 \%$, and higher usage of RAS inhibitors (76.9\%) and $\beta$ blockers (80.1\%). In the present study, the use of DOACs was associated with lower all-cause mortality than VKAs and nonanticoagulant in HHF patient with AF. HF is reportedly associated with poor control of VKAs [28]. It has been recently reported that DOACs, compared with VKAs, is associated with lower prevalence of major bleeding [14] and avoidance of anemia, as well as lower occurrence of kidney injury [29], acute coronary syndrome [30] and HF [30]. These backgrounds may be associated with lower all-cause mortality in HHF patients with AF.

\section{Study strengths and limitations}

Our study has several strengths, and differs from previous studies [7-9, 14]. For instance, the present study is the first to show the effect of DOACs on reducing allcause mortality in HHF patients with AF considering the clinical background, including $\mathrm{CHA}_{2} \mathrm{DS}_{2}$-Vasc and HAS-BLED scores, other co-morbidities, and pharmacotherapies. In addition, the diagnosis of HF and causes of death were accurately made by our experienced cardiologists, based on the Framingham criteria. Furthermore, there were no patients who dropped out.

There are some potential limitations. Firstly, our study was a observational study at a single institution, and thus the numbers of subjects were relatively small. Although the PS analyses are useful, they are inherently limited by the number and accuracy of the variables evaluated. Importantly, we cannot rule out residual confounding from unknown or unmeasured variables. There might be potential bias and although we analyzed present data using multivariate Cox proportional hazard regression analyses and PS analyses to minimize selection bias under consideration of multiple confounding factors, the effects of the differences in clinical background between the groups might not be completely adjusted. Secondly, we have assessed this study using only variables on hospitalization, without consideration of changes in medical parameters and post-discharge treatment. There might be some cross over in medication during follow up. Thirdly, TTR of VKAs was insufficient; however, TTR in this study was higher than those of previous studies [7-9]. Finally, the differences of each DOAC were not considered in this study. For these reasons, the results of our study should be viewed as preliminary. Hence, further clinical trials for HHF patients with concomitant AF using DOACs are required with a larger population and/or randomization.

\section{Conclusion}

The appropriate use of anticoagulants in HHF patients with AF is important, and DOACs potentially improve all-cause mortality in such patients.

\section{Additional file}

Additional file 1: Table S1. The clinical features of DOACs group and VKAs group in the post-matched cohort are summarized in Table S1. (DOC $69 \mathrm{~kb}$ )

\section{Abbreviations \\ AF: Atrial fibrillation; BNP: B-type natriuretic peptide; Cl: Confidence interval; CKD: Chronic kidney disease; DOACs: Direct oral anticoagulants; HF: Heart failure; HHF: Hospitalized heart failure; HR: Hazard ratio; LVEF: Left ventricular ejection fraction; PS: Propensity score; RR: Relative risk; SR: Sinus rhythm; TTR: Time in therapeutic range; VKAs: Vit K antagonists}

\section{Acknowledgements}

The authors acknowledge the efforts of Dr. Tetsuya Ohira (Department of Epidemiology) for his invaluable advice on medical statistics, as well as Ms. Kumiko Watanabe and Ms. Hitomi Kobayashi for their outstanding technical assistance, and Dr. Shunsuke Watanabe, Dr. Yuki Kanno, Dr. Akihiko Sato, Dr. Tetsuro Yokokawa, Dr. Shunsuke Miura, Dr. Satoshi Abe, Dr. Atsushi Kobayashi, Dr. Takayoshi Yamaki, Dr. Hiroyuki Kunii, Dr. Kazuhiko Nakazato, Dr. Hitoshi Suzuki and Dr. Shu-ichi Saitoh for their acquisition of data.

\section{Funding}

Not applicable

\section{Availability of data and materials}

The datasets used and/or analysed during the current study are available from the corresponding author on reasonable request.

\section{Authors' contributions}

AY, SS and YT, making article, drafting the article and conception of this study; YS, TS analysis and interpretation of data; $\mathrm{MO}$, and $\mathrm{YT}$ revising the article critically for important intellectual content. All authors read and approved the final manuscript.

Ethics approval and consent to participate

The study protocol conforms to the ethical guidelines of the 1975 Declaration of Helsinki as reflected in a prior approval by the institution's human research committee (Fukushima Medical University). Written informed consent was obtained from each subject. 


\section{Competing interests}

The authors declare that they have no competing interests

\section{Publisher's Note}

Springer Nature remains neutral with regard to jurisdictional claims in published maps and institutional affiliations.

Received: 14 July 2017 Accepted: 16 January 2018

Published online: 25 January 2018

\section{References}

1. Savelieva I, John Camm A. Atrial fibrillation and heart failure: natural history and pharmacological treatment. Europace. 2004;5(Suppl 1):S5-19.

2. Santhanakrishnan R, Wang N, Larson MG, Magnani JW, McManus DD, Lubitz SA, Ellinor PT, Cheng S, Vasan RS, Lee DS, et al. Atrial fibrillation begets heart failure and vice versa: temporal associations and differences in preserved versus reduced ejection fraction. Circulation. 2016;133(5):484-92.

3. Wang TJ, Larson MG, Levy D, Vasan RS, Leip EP, Wolf PA, D'Agostino RB, Murabito JM, Kannel WB, Benjamin EJ. Temporal relations of atrial fibrillation and congestive heart failure and their joint influence on mortality: the Framingham heart study. Circulation. 2003:107(23):2920-5.

4. Maisel WH, Stevenson LW. Atrial fibrillation in heart failure: epidemiology, pathophysiology, and rationale for therapy. Am J Cardiol. 2003;91(6A):2D-8D.

5. Lip GY, Laroche C, loachim PM, Rasmussen LH, Vitali-Serdoz L, Petrescu L, Darabantiu D, Crijns HJ, Kirchhof P, Vardas P, et al. Prognosis and treatment of atrial fibrillation patients by European cardiologists: one year follow-up of the EURObservational research Programme-Atrial fibrillation general registry pilot phase (EORP-AF pilot registry). Eur Heart J. 2014;35(47):3365-76.

6. Reinier K, Marijon E, Uy-Evanado A, Teodorescu C, Narayanan K, Chugh H, Gunson K, Jui J, Chugh SS. The association between atrial fibrillation and sudden cardiac death: the relevance of heart failure. JACC Heart failure. 2014;2(3):221-7.

7. Ferreira J, Ezekowitz MD, Connolly SJ, Brueckmann M, Fraessdorf M, Reilly PA, Yusuf S, Wallentin L, Investigators R-L. Dabigatran compared with warfarin in patients with atrial fibrillation and symptomatic heart failure: a subgroup analysis of the RE-LY trial. Eur J Heart Fail. 2013;15(9):1053-61.

8. McMurray JJ, Ezekowitz JA, Lewis BS, Gersh BJ, van Diepen S, Amerena J, Bartunek J, Commerford P, Oh BH, Harjola VP, et al. Left ventricular systolic dysfunction, heart failure, and the risk of stroke and systemic embolism in patients with atrial fibrillation: insights from the ARISTOTLE trial. Circ Heart Fail. 2013;6(3):451-60.

9. van Diepen S, Hellkamp AS, Patel MR, Becker RC, Breithardt G, Hacke W, Halperin JL, Hankey GJ, Nessel CC, Singer DE, et al. Efficacy and safety of rivaroxaban in patients with heart failure and nonvalvular atrial fibrillation: insights from ROCKET AF. Circ Heart Fail. 2013;6(4):740-7.

10. Camm AJ, Lip GY, De Caterina R, Savelieva I, Atar D, Hohnloser SH, Hindricks G, Kirchhof P, Guidelines ESCCFP. Focused update of the ESC guidelines for the management of atrial fibrillation: an update of the 2010 ESC guidelines for the management of atrial fibrillation. Developed with the special contribution of the European heart rhythm association. Eur Heart J 2012 2012:33(21):2719-47.

11. Okumura K, Hori M, Tanahashi N, John Camm A. Special considerations for therapeutic choice of non-vitamin $\mathrm{K}$ antagonist oral anticoagulants for Japanese patients with nonvalvular atrial fibrillation. Clin Cardiol. 2017:40(2):126-31.

12. Ruff $C T$, Giugliano RP, Braunwald E, Hoffman EB, Deenadayalu N, Ezekowitz MD, Camm AJ, Weitz Jl, Lewis BS, Parkhomenko A, et al. Comparison of the efficacy and safety of new oral anticoagulants with warfarin in patients with atrial fibrillation: a meta-analysis of randomised trials. Lancet. 2014;383(9921):955-62.

13. Albert NM. Use of novel oral anticoagulants for patients with atrial fibrillation: systematic review and clinical implications. Heart Lung. 2014;43(1):48-59.

14. Xiong Q, Lau YC, Senoo K, Lane DA, Hong K, Lip GY. Non-vitamin K antagonist oral anticoagulants (NOACs) in patients with concomitant atrial fibrillation and heart failure: a systemic review and meta-analysis of randomized trials. Eur J Heart Fail. 2015;17(11):1192-200.

15. Vaduganathan M, Butler J, Roessig L, Fonarow GC, Greene SJ, Metra M Cotter G, Kupfer S, Zalewski A, Sato N, et al. Clinical trials in hospitalized heart failure patients: targeting interventions to optimal phenotypic subpopulations. Heart Fail Rev. 2015;20(4):393-400.

16. Ambrosy AP, Fonarow GC, Butler J, Chioncel O, Greene SJ, Vaduganathan M, Nodari S, Lam CS, Sato N, Shah AN, et al. The global health and economic burden of hospitalizations for heart failure: lessons learned from hospitalized heart failure registries. J Am Coll Cardiol. 2014:63(12):1123-33.

17. Fonarow GC, Albert NM, Curtis AB, Gheorghiade M, Heywood JT, Liu Y, Mehra MR, O'Connor CM, Reynolds D, Walsh MN, et al. Associations between outpatient heart failure process-of-care measures and mortality. Circulation. 2011;123(15):1601-10.

18. Camm AJ, Accetta G, Ambrosio G, Atar D, Bassand JP, Berge E, Cools F, Fitzmaurice DA, Goldhaber SZ, Goto S, et al. Evolving antithrombotic treatment patterns for patients with newly diagnosed atrial fibrillation. Heart. 2017;103(4):307-14.

19. McKee PA, Castelli WP, McNamara PM, Kannel WB. The natural history of congestive heart failure: the Framingham study. N Engl J Med. 1971;285(26):1441-6.

20. von Elm E, Altman DG, Egger M, Pocock SJ, Gotzsche PC, Vandenbroucke $J P$, Initiative S. Strengthening the reporting of observational studies in epidemiology (STROBE) statement: guidelines for reporting observational studies. BMJ. 2007;335(7624):806-8.

21. D'Agostino RB Jr. Propensity score methods for bias reduction in the comparison of a treatment to a non-randomized control group. Stat Med. 1998;17(19):2265-81.

22. Heinze $G$, Juni P. An overview of the objectives of and the approaches to propensity score analyses. Eur Heart J. 2011;32(14):1704-8.

23. Austin PC. A critical appraisal of propensity-score matching in the medical literature between 1996 and 2003. Stat Med. 2008:27(12):2037-49.

24. Connolly SJ, Ezekowitz MD, Yusuf S, Eikelboom J, Oldgren J, Parekh A, Pogue J, Reilly PA, Themeles E, Varrone J, et al. Dabigatran versus warfarin in patients with atrial fibrillation. N Engl J Med. 2009;361(12):1139-51.

25. Patel MR, Mahaffey KW, Garg J, Pan G, Singer DE, Hacke W, Breithardt G, Halperin JL, Hankey GJ, Piccini JP, et al. Rivaroxaban versus warfarin in nonvalvular atrial fibrillation. N Engl J Med. 2011;365(10):883-91.

26. Granger CB, Alexander JH, McMurray JJ, Lopes RD, Hylek EM, Hanna M, Al-Khalidi HR, Ansell J, Atar D, Avezum A, et al. Apixaban versus warfarin in patients with atrial fibrillation. N Engl J Med. 2011;365(11):981-92.

27. Giugliano RP, Ruff CT, Braunwald E, Murphy SA, Wiviott SD, Halperin JL, Waldo AL, Ezekowitz MD, Weitz J, Spinar J, et al. Edoxaban versus warfarin in patients with atrial fibrillation. N Engl J Med. 2013;369(22):2093-104.

28. Nelson WW, Choi JC, Vanderpoel J, Damaraju CV, Wildgoose P, Fields LE, Schein JR. Impact of co-morbidities and patient characteristics on international normalized ratio control over time in patients with nonvalvular atrial fibrillation. Am J Cardiol. 2013;112(4):509-12.

29. Chan YH, Yeh YH, See LC, Wang CL, Chang SH, Lee HF, Wu LS, Tu HT, Kuo CT. Acute kidney injury in Asians with Atrial fibrillation treated with Dabigatran or Warfarin. J Am Coll Cardiol. 2016;68(21):2272-83.

30. Vaughan Sarrazin MS, Jones M, Mazur A, Cram P, Ayyagari P, Chrischilles E. Cost of hospital admissions in Medicare patients with Atrial fibrillation taking Warfarin, Dabigatran, or rivaroxaban. J Am Coll Cardiol. 2017:69(3):360-2.

\section{Submit your next manuscript to BioMed Central and we will help you at every step:}

- We accept pre-submission inquiries

- Our selector tool helps you to find the most relevant journal

- We provide round the clock customer support

- Convenient online submission

- Thorough peer review

- Inclusion in PubMed and all major indexing services

- Maximum visibility for your research

Submit your manuscript at www.biomedcentral.com/submit
) Biomed Central 